\title{
Non-radial oscillations in the red giant HR 7349 measured by CoRoT*
}

\author{
F. Carrier ${ }^{1}$, J. De Ridder ${ }^{1}$, F. Baudin ${ }^{2}$, C. Barban ${ }^{3}$, A. P. Hatzes ${ }^{4}$, S. Hekker ${ }^{5,6,1}$, T. Kallinger ${ }^{7,8}$, A. Miglio ${ }^{9}$,

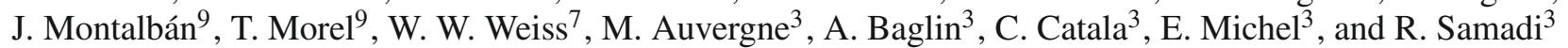 \\ 1 Instituut voor Sterrenkunde, Katholieke Universiteit Leuven, Celestijnenlaan 200D, 3001 Leuven, Belgium \\ e-mail: fabien@ster.kuleuven.be \\ 2 Institut d'Astrophysique Spatiale, Campus d'Orsay, 91405 Orsay, France \\ 3 LESIA, UMR8109, Université Pierre et Marie Curie, Université Denis Diderot, Observatoire de Paris, 92195 Meudon, France \\ 4 Thüringer Landessternwarte, 07778 Tautenburg, Germany \\ 5 University of Birmingham, School of Physics and Astronomy, Edgbaston, Birmingham B15 2TT, UK \\ 6 Royal Observatory of Belgium, Ringlaan 3, 1180 Brussels, Belgium \\ 7 Institute for Astronomy, University of Vienna, Türkenschanzstrasse 17, 1180 Vienna, Austria \\ 8 Department of Physics and Astronomy, University of British Columbia, 6224 Agricultural Road, Vancouver, BC V6T 1Z1, Canada \\ 9 Institut d'Astrophysique et de Géophysique de l'Université de Liège, Allée du 6 Août 17, 4000 Liège, Belgium
}

Received 23 June 2009 / Accepted 7 October 2009

\section{ABSTRACT}

\begin{abstract}
Context. Convection in red giant stars excites resonant acoustic waves whose frequencies depend on the sound speed inside the star, which in turn depends on the properties of the stellar interior. Therefore, asteroseismology is the most robust available method for probing the internal structure of red giant stars.

Aims. Solar-like oscillations in the red giant HR 7349 are investigated.

Methods. Our study is based on a time series of 380760 photometric measurements spread over 5 months obtained with the CoRoT satellite. Mode parameters were estimated using maximum likelihood estimation of the power spectrum.

Results. The power spectrum of the high-precision time series clearly exhibits several identifiable peaks between 19 and $40 \mu \mathrm{Hz}$ showing regularity with a mean large and small spacing of $\Delta v=3.47 \pm 0.12 \mu \mathrm{Hz}$ and $\delta v_{02}=0.65 \pm 0.10 \mu \mathrm{Hz}$. Nineteen individual modes are identified with amplitudes in the range from 35 to $115 \mathrm{ppm}$. The mode damping time is estimated to be $14.7_{-2.9}^{+4.7}$ days.
\end{abstract}

Key words. stars: variables: general - stars: oscillations - stars: interiors - stars: individual: HR 7349

\section{Introduction}

The analysis of the oscillation spectrum provides an unrivaled method for probing the stellar internal structure because the frequencies of these oscillations depend on the sound speed inside the star, which in turn depends on the density, temperature, gas motion, and other properties of the stellar interior. High-precision spectrographs have acquired data yielding to a rapidly growing list of solar-like oscillation detections in mainsequence and giant stars (see e.g., Bedding \& Kjeldsen 2007; Carrier et al. 2008). In a few years, we have moved from ambiguous detections to firm measurements. Among these, only a few are related to red giants, e.g., $\xi$ Hya, Frandsen et al. (2002), $\epsilon$ Oph, De Ridder et al. (2006), and $\eta$ Ser, Barban et al. (2004). The reason is that longer and almost uninterrupted time series are needed to characterize the oscillations in red giants, because of longer oscillation periods than main-sequence stars, and long observing runs are difficult to obtain using high-accuracy spectrographs.

The CoRoT (COnvection ROtation and planetary Transits) satellite (Baglin 2006) is perfect for this purpose because it can provide these data for a large number of stars simultaneously. The CoRoT satellite continuously collects white-light high-precision photometric observations for 10 bright stars in the so-called seismofield, as well as 3-color photometry for

* The CoRoT space mission has been developed and is operated by CNES, with the contribution of Austria, Belgium, Brazil, ESA, Germany and Spain. thousands of relatively faint stars in the so-called exofield. The primary motivation for acquiring this second set of data is to detect planetary transits, but the data are also well suited to asteroseismic investigations. De Ridder et al. (2009) unambiguously detected long-lifetime non-radial oscillations in red giant stars in the exofield data of CoRoT, which is an important breakthrough for asteroseismology. Indeed, observations from either the ground or other satellites have been unable to confirm the existence of non-radial modes and determine a clear value of the mode lifetime. Hekker et al. (2009) presented a more detailed classification of the red giants observed by CoRoT.

HR 7349 (HD 181907) is a bright equatorial G8 giant star $(V=5.82)$ that is an excellent target for asteroseismology. This star was selected as a secondary target during the first long run of the CoRoT mission. In this paper, we thus report on photometric CoRoT observations of HR 7349 resulting in the detection and identification of p-mode oscillations. The non-asteroseismic observations are presented in Sect. 2, the CoRoT data and frequency analysis in Sects. 3 and 4, and the conclusions are given in Sect. 5.

\section{Fundamental parameters}

\subsection{Effective temperature and chemical composition}

We used the line analysis code MOOG, Kurucz models, and a high-resolution FEROS spectrum obtained in June 2007 to carry out an LTE abundance study of HR 7349. The effective 
temperature and surface gravity were estimated from the excitation and ionization equilibrium of a set of iron lines taken from the line list of Hekker \& Meléndez (2007). We obtain $T_{\text {eff }}=4790 \pm 80 \mathrm{~K}$ and $[\mathrm{Fe} / \mathrm{H}]=-0.08 \pm 0.10$ dex, while the abundance pattern of the other elements with respect to $\mathrm{Fe}$ is solar within the errors. The full results of the abundance analysis will be reported elsewhere (Morel et al., in preparation). We also determined a photometric temperature given by the relation in Alonso et al. (1999) using the dereddened color index $(B-V)$ (see Sect. 2.2) and found $4704 \pm 110 \mathrm{~K}$, which agrees with the spectroscopic value. We finally adopt a weighted-mean temperature of $4760 \pm 65 \mathrm{~K}$.

\subsection{Luminosity}

Even for such a bright star, the interstellar extinction in the direction of the Galactic center is not negligible. From the HIPPARCOS parallax $\Pi=9.64 \pm 0.34$ mas (van Leeuwen 2007) and the value of $(B-V)=1.093$ in the HIPPARCos catalog, an absorption of $A_{V}=0.185 \mathrm{mag}$ is derived for the region of the star (Arenou et al. 1992), which corresponds to an $E_{B-V}=0.052$. Combining the magnitude $V=5.809 \pm 0.004$ (Geneva photometry, Burki et al. 2008), the HIPPARCos parallax, the solar absolute bolometric magnitude $M_{\mathrm{bol}, \odot}=4.746$ (Lejeune et al. 1998), and the mean bolometric corrections $\mathrm{BC}=-0.40 \pm 0.04 \mathrm{mag}$ $(\mathrm{BC}=-0.42 \pm 0.06 \mathrm{mag}$ according to the calibration of Flower, 1996 and $\mathrm{BC}=-0.38 \pm 0.05 \mathrm{mag}$ by Alonso et al. 1999), we find a luminosity for HR 7349 of $L=69 \pm 6 L_{\odot}$.

\subsection{Rotational velocity}

We determined the rotational velocity of the star by means of a spectrum taken with the spectrograph CORALIE installed on the 1.2-m Swiss telescope, ESO La Silla, Chile. According to the calibration of Santos et al. (2002), we determined a $v \sin i=1.0 \pm 1.0 \mathrm{~km} \mathrm{~s}^{-1}$. For this small value of the projected rotational velocity, we do not expect to see any split modes in the power spectrum.

\subsection{Large spacing estimation}

An estimation of the mass of HR 7349 may be obtained by matching evolutionary tracks to the $L-T_{\text {eff }}$ error box in the HR diagram. However, in the red giant part of the HR diagram, this determination is not robust at all. Assuming a mass of between 0.8 and $3 M_{\odot}$ and scaling from the solar case (Kjeldsen $\&$ Bedding 1995), a large frequency spacing of $2.8-5.5 \mu \mathrm{Hz}$ is expected.

\section{CoRoT observations}

HR 7349 was observed with the CoRoT satellite for 5 consecutive months. CoRoT was launched on 2006 December 27 from Baikonur cosmodrome on a Soyuz Fregat II-1b launcher. The raw photometric data acquired with CoRoT were reduced by the CoRoT team. A detailed description of how photometric data are extracted for the seismology field was presented in Baglin (2006). A summary can be found in Appourchaux et al. (2008).

In the seismofield, CoRoT obtains one measurement every $32 \mathrm{~s}$. The observations lasted for 156.64 days from May 11th to October 15th 2007. The light curve shows near-continuous coverage over the 5 months, with only a small number of gaps due mainly to the passage of CoRoT across the South Atlantic

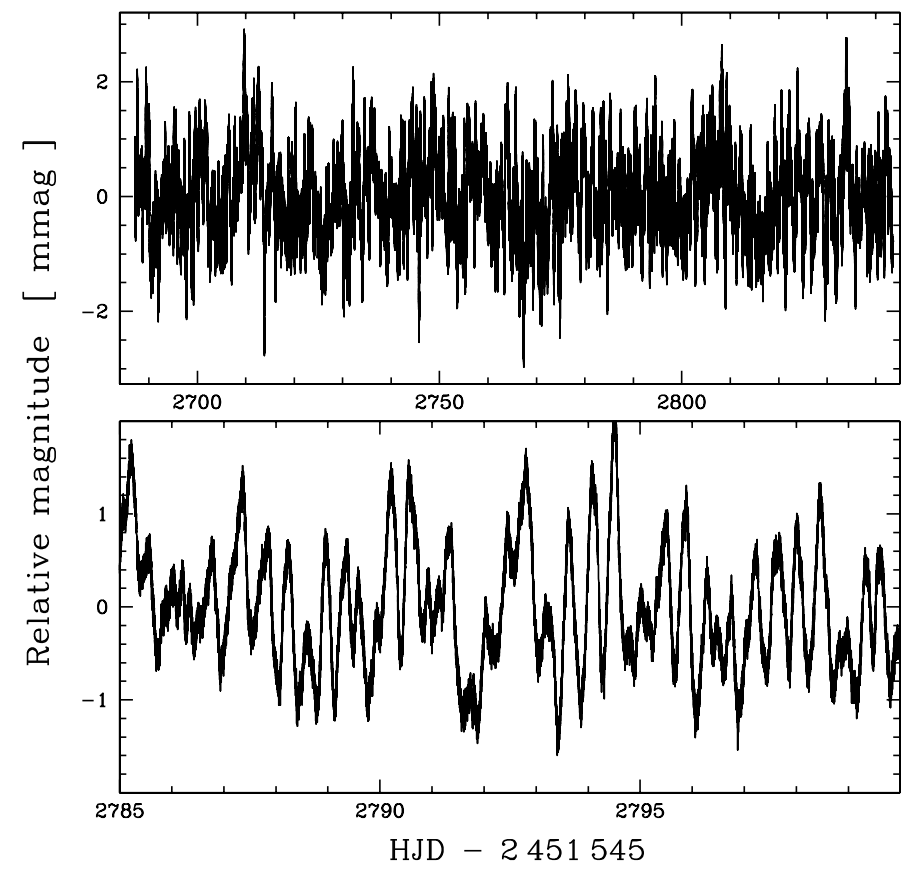

Fig. 1. The total CoRoT light curve (top) and a zoom (bottom) of HR 7349. This light curve is detrended with a polynomial fit (order 8), which only affects frequencies below $6 \mu \mathrm{Hz}$. A periodicity of about $8.5 \mathrm{~h}$ can be seen in the zoom, corresponding to oscillation modes close to $30 \mu \mathrm{Hz}$.

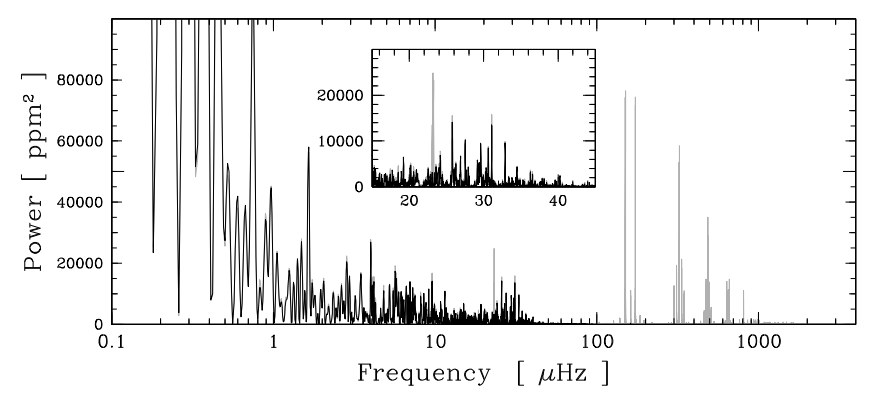

Fig. 2. Power spectra of the original data (grey) and interpolated data (black). The range of the oscillation is zoomed in the inset. The interpolation drastically reduces the amplitude of aliases, in particular the one at $23 \mu \mathrm{Hz}$.

Anomaly. These short gaps were filled by suitable interpolation (Baglin 2006), without any influence on the mode extraction because it only affects the amplitude of frequencies far above the oscillation range of our target (see Fig. 2). The duty cycle for HR 7349 before interpolation was $90 \%$. For the frequency analysis (see Sect. 4), the light curve was detrended with a polynomial fit to remove the effect of the aging of the CCDs (see Auvergne et al. 2009). This detrending has no consequence on the amplitude or frequency of oscillation modes, since it only affects the power spectrum for frequencies lower than $6 \mu \mathrm{Hz}$. The light curve shows variations of a timescale of 8-9 h and peakto-peak amplitudes of $1-3$ mmag (see Fig. 1). This signal is a superposition of tens of smaller modes with similar periods (see Sect. 4).

\section{Frequency analysis}

\subsection{Noise determination}

We computed the power spectra of CoRoT light curves with both gaps and interpolated points (see Sect. 3). The resulting 


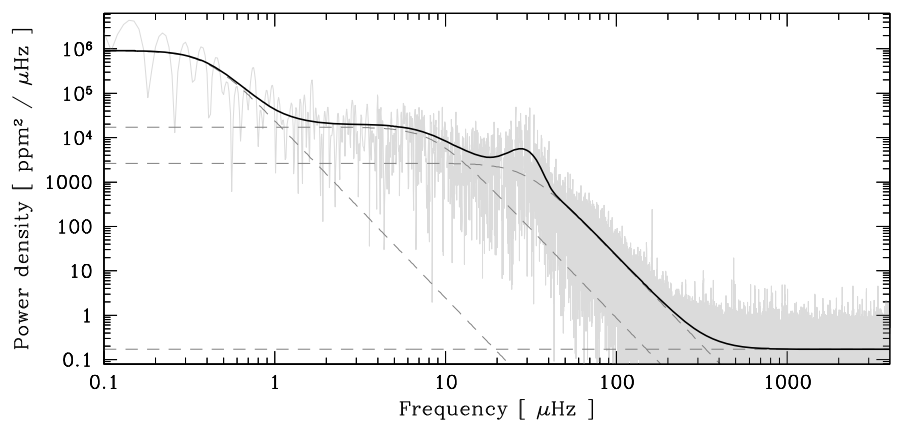

Fig. 3. Power density spectrum of the photometric time series of HR 7349 and a multi-component function (black line) fitted to the heavily smoothed power density spectrum. The function is the superposition of three power-law components (dashed lines), white noise (horizontal dashed line) and a power excess hump approximated by a Gaussian function.

power spectra are quasi-identical, the interpolation not affecting the oscillations but suppressing the aliases (the most important of which lies at $23 \mu \mathrm{Hz}$ ). We thus analyze the interpolated time series with negligible alias amplitudes. The time base of the observations gives a formal resolution of $0.07 \mu \mathrm{Hz}$. The power (density) spectrum of the time series, shown in Figs. 3 and 4, exhibits a series of peaks between 20 and $40 \mu \mathrm{Hz}$, exactly where the solar-like oscillations are expected for this star. The power density spectrum is independent of the observing window: this is achieved by multiplying the power by the effective length of the observing run (we have to divide by the resolution for equidistant data), which is calculated to be the reciprocal of the area beneath the spectral window in power (Kjeldsen et al. 2005). We note that to obtain the same normalisation as in Baudin et al. (2005), we multiply the power by the effective length of the observation divided by four. Typically for such a power spectrum, the noise has two components:

- at high frequencies it is flat, indicative of the Poisson statistics of photon noise;

- towards the lowest frequencies, the power scales inversely with frequency, as expected for instrumental instabilities and noise of stellar origin like granulation.

For the Sun, it is common practice to model the background signal with power laws to allow accurate measurements of solar oscillation frequencies and amplitudes (Harvey 1985; Andersen et al. 1998; Aigrain et al. 2004). To study this "noise", we compute the power density spectrum shown in Fig. 3 and fit a smoothed version of this spectrum with a sum of $N$ power laws

$P(v)=\sum_{i=1}^{N} P_{i}=\sum_{i=1}^{N} \frac{A_{i}}{1+\left(B_{i} v\right)^{C_{i}}}$,

where this number $N$ depends on the frequency coverage, $v$ is the frequency, $A_{i}$ is the amplitude of the $i$ th component, $B_{i}$ is its characteristic timescale, and $C_{i}$ is the slope of the power law. For a given component, the power remains approximately constant on timescales longer than $B_{i}$, and drops off for shorter timescales. Each power law corresponds to a separate class of physical phenomena, occurring on a different characteristic timescale, and corresponding to different physical structures on the surface of the star. In our case, we fixed the slope to 4, which is a typical value for the Sun (Aigrain et al. 2004; Michel et al. 2009). Moreover, this value allows us to fit our power density spectrum well.

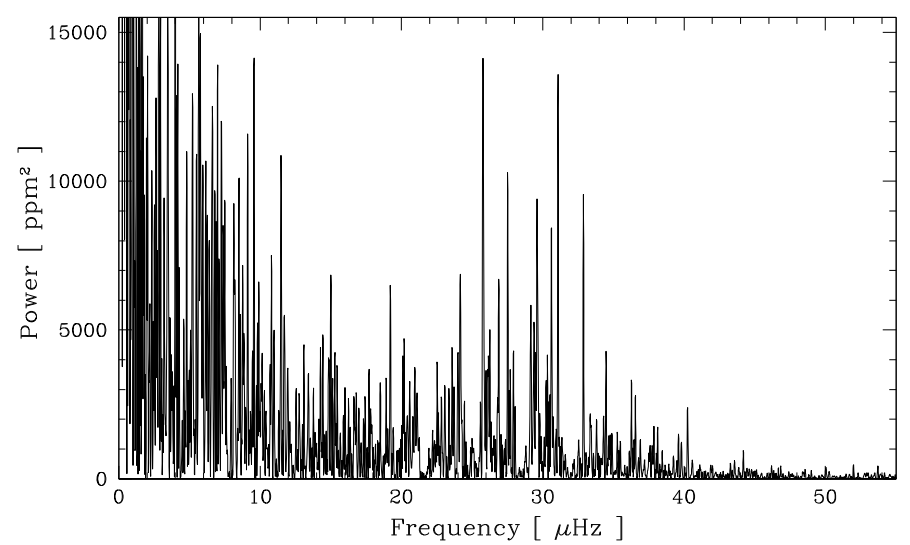

Fig. 4. Power spectrum of the CoRoT observations of HR 7349. Only a polynomial fit was removed from the original data.

To model the power density spectrum, we added a white noise $P_{n}$ and a power excess hump produced by the oscillations, which was approximated by a Gaussian function

$P(v)=\sum_{i=1}^{N} \frac{A_{i}}{1+\left(B_{i} v\right)^{C_{i}}}+P_{n}+P_{g} \mathrm{e}^{-\left(v_{\max }-v\right)^{2} /\left(2 \sigma^{2}\right)}$

The number of components $N$ is determined iteratively: we first made a single component fit, and additional components were then added until they no longer improved the fit. In our case, we limited the number of components to three. We note that for this star, part of the low frequency variation is caused by the aging of the CCD. The timescales for the different noise sources $\left(B_{i}\right)$ are $3.6 \times 10^{6}, 6.9 \times 10^{4}$, and $1.1 \times 10^{4} \mathrm{~s}$. The noise at high frequencies $P_{n}$ is only $0.17 \mathrm{ppm}^{2} / \mu \mathrm{Hz}$ and the oscillations are centered on $28.2 \mu \mathrm{Hz}$.

\subsection{Search for a comb-like pattern}

In solar-like stars, p-mode oscillations are expected to produce a characteristic comb-like structure in the power spectrum with mode frequencies $v_{n, \ell}$ reasonably well approximated by the asymptotic relation (Tassoul 1980):

$v_{n, \ell} \approx \Delta v\left(n+\frac{\ell}{2}+\epsilon\right)-\ell(\ell+1) D_{0}$

Here $D_{0}$ (which equals $\frac{1}{6} \delta v_{02}$ if the asymptotic relation holds exactly) and $\epsilon$ are sensitive to the sound speed near the core and in the surface layers, respectively. The quantum numbers $n$ and $\ell$ correspond to the radial order and the angular degree of the modes, and $\Delta v$ and $\delta v_{02}$ are the large and small spacings. We note that a giant star such as HR 7349 is expected to show substantial deviations from its regular comb-like structure described above (Christensen-Dalsgaard 2004). This is because some mode frequencies, except for $\ell=0$, may be shifted from their usual regular spacing by avoided crossings with gravity modes in the stellar core (also called "mode bumping") (see e.g., Christensen-Dalsgaard et al. 1995; and Fernandes \& Monteiro 2003). We must keep the possibility of these mixed modes in mind when attempting to identify oscillation modes in the power spectrum. Moreover, the ratio of lifetime to oscillation periods is usually far smaller for red giants (because of their longer periods) than for solar-like stars, which can complicate the mode detection (see e.g., Stello et al. 2006; Tarrant et al. 2008). 


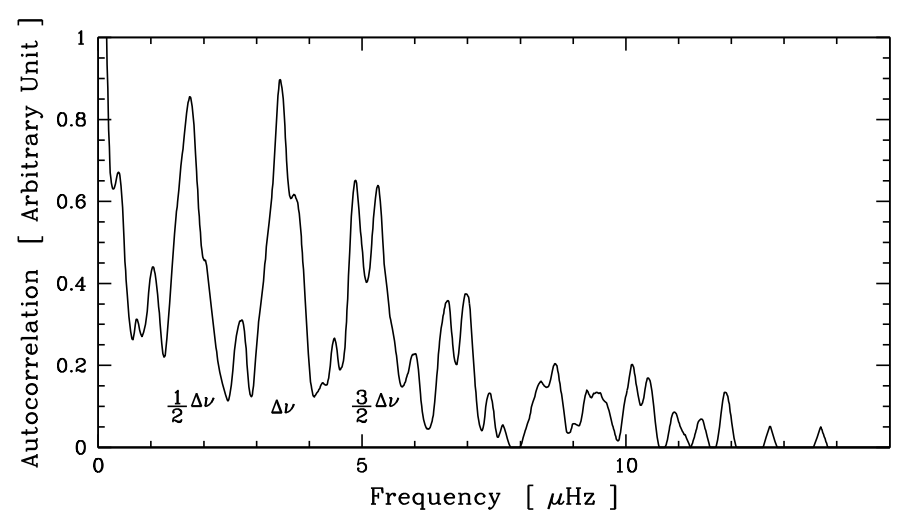

Fig. 5. Autocorrelation of the slightly smoothed power spectrum. The large spacing, as well as separations between $\ell=0$ and $\ell=1$ modes are clearly present.

The first step is to measure the large spacing that should appear at least between radial modes. The power spectrum is autocorrelated to search for periodicity. Each peak of this autocorrelation (see Fig. 5) corresponds to a structure present in the power spectrum. One of the three strong groups of peaks at about 1.7, 3.5 , and $5.2 \mu \mathrm{Hz}$ should correspond to the large spacing. By visually inspecting the power spectrum, the value of about $3.5 \mu \mathrm{Hz}$ is adopted as the large separation, the two others are spacings between the $\ell=0$ and $\ell=1$ modes. This large separation value is in good agreement with the scaled value from the solar case (see Sect. 2.4).

\subsection{Extraction of mode parameters}

The power spectra in Figs. 6 and 7 clearly exhibit a regularity that allows us to identify $\ell=0$ to $\ell=2$ modes. Each mode consists of several peaks, which is the clear signature of a finite lifetime shorter than the observing time span. In order to determine the mode frequencies, as well as amplitude and lifetime of the modes, we fitted the power spectrum using a maximum likelihood estimation (MLE) method. MLE has been applied widely in the helioseismic community (e.g., see Schou 1992; Appourchaux et al. 1998; and Chaplin et al. 2006). Our program uses the IDL routines developed by Appourchaux (1998). This method has already been used with success for the red giant $\epsilon$ Oph (Barban et al. 2007).

The modelled power spectrum for a series of $M$ oscillation modes, $P\left(v_{k}\right)$, is

$P\left(v_{k}\right)=\sum_{n=1}^{M}\left(H_{n} \frac{1}{1+\left(\frac{2\left(v_{k}-v_{n}\right)^{2}}{\Gamma_{n}}\right)}\right)+B$,

where $H_{n}$ is the height of the Lorentzian profile, $v_{n}$ is the oscillation mode frequency, $\Gamma_{n}$ is the mode line-width, and $B$ is the background noise. The fit was performed on the non-oversampled power spectrum to minimize the interdependency of the points. The quantity that was minimized is

$L=\sum_{k=1}^{K}\left(\ln P\left(v_{k}\right)+\frac{P_{\mathrm{obs}}\left(v_{k}\right)}{P\left(v_{k}\right)}\right)$,

where $K$ is the number of bins, i.e., the number of Fourier frequencies.

Only a few peaks belong to a given mode, indicating a lifetime longer than 10 days. It is thus also difficult to derive the

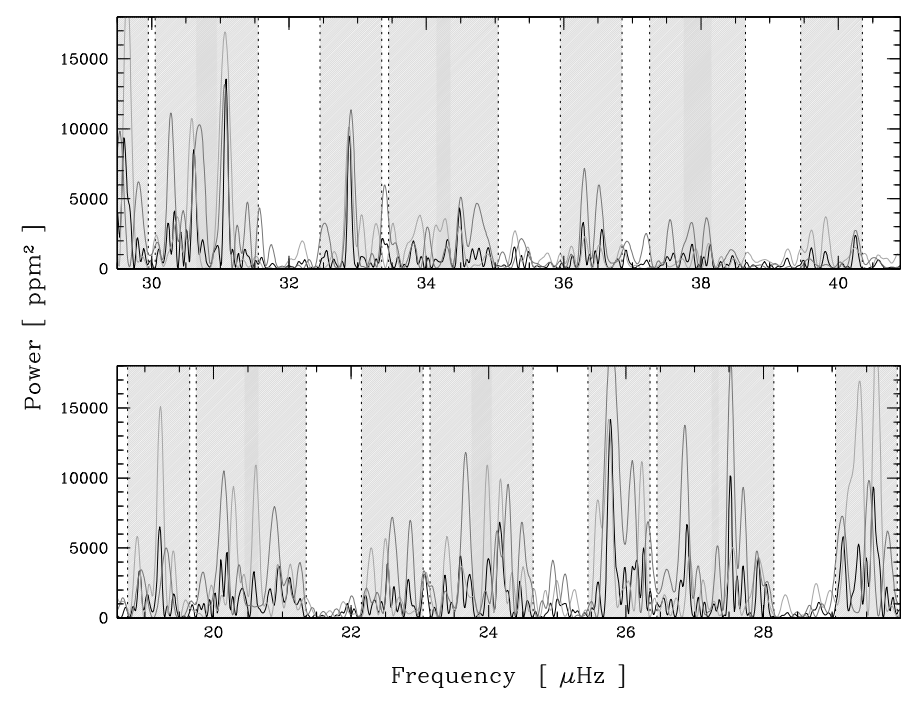

Fig. 6. Power spectra of the whole dataset (black) and of the two halflong subsets (grey). The frequencies of the oscillation peaks change from one subset to the other, which is a sign of a finite lifetime. The initial guess for the identification of modes are indicated by shaded regions: these regions are regularly spaced in agreement with the large separation deduced from the autocorrelation. Every second region has a structure more simple and narrow, corresponding to our identification of $\ell=1$ modes, the others are more complex and wide and correspond to $\ell=0$ and 2 modes.

correct Lorentzian shape for each mode. Therefore some parameters were fixed to avoid incorrect parameter determinations:

- the noise was determined independently of the MLE method (see Sect. 4.1);

- since no difference between the shapes of modes of different degree $\ell$ is detected and the value of $v \sin i$ is extremely small, resulting in an expected rotational splitting value smaller than $0.03 \mu \mathrm{Hz}$, we assumed that it is zero;

- when fitting all modes without fixing the width of the Lorentzian envelope, we clearly saw that the fit was not robust enough to provide an accurate determination of all mode parameters. The method was thus first to find a mean value for the Lorentzian width and to fix this mean value for all modes. The mean width of the Lorentzian is obtained by individually fitting all modes and by taking their mean, rejecting the values that obviously correspond to a poor fit. The determined mean is $0.25 \pm 0.06 \mu \mathrm{Hz}$, which corresponds to a mode lifetime of $14.7_{-2.9}^{+4.7} \mathrm{~d}$. The use of sub-series show the stochastic nature of the mode excitation caused by the different fine structure of peaks in each spectrum, and give an indication of their width. We note that we also checked that no significant width difference was found for modes with different degrees $\ell$ (by comparing their mode-width means).

All modes were then fitted with fixed parameters as deduced above. We note that the initial guess values are indicated by shaded regions in Fig. 6. These regions are already in good agreement with a regular spacing between modes. Every second region has a structure that is more simple and narrow, corresponding to our identification of $\ell=1$ modes, the others are more complex and wide. In this last case, we needed to fit two modes per region to reproduce the power spectrum (which correspond to $\ell=0$ and 2 modes). At $38 \mu \mathrm{Hz}$, the signal-to-noise ratio is far smaller and it becomes more difficult to differentiate between modes: for the sake of homogeneity, we also decided to fit two modes in this region. The frequencies of these last 
F. Carrier et al.: Non-radial oscillations in the red giant HR 7349 measured by CoRoT

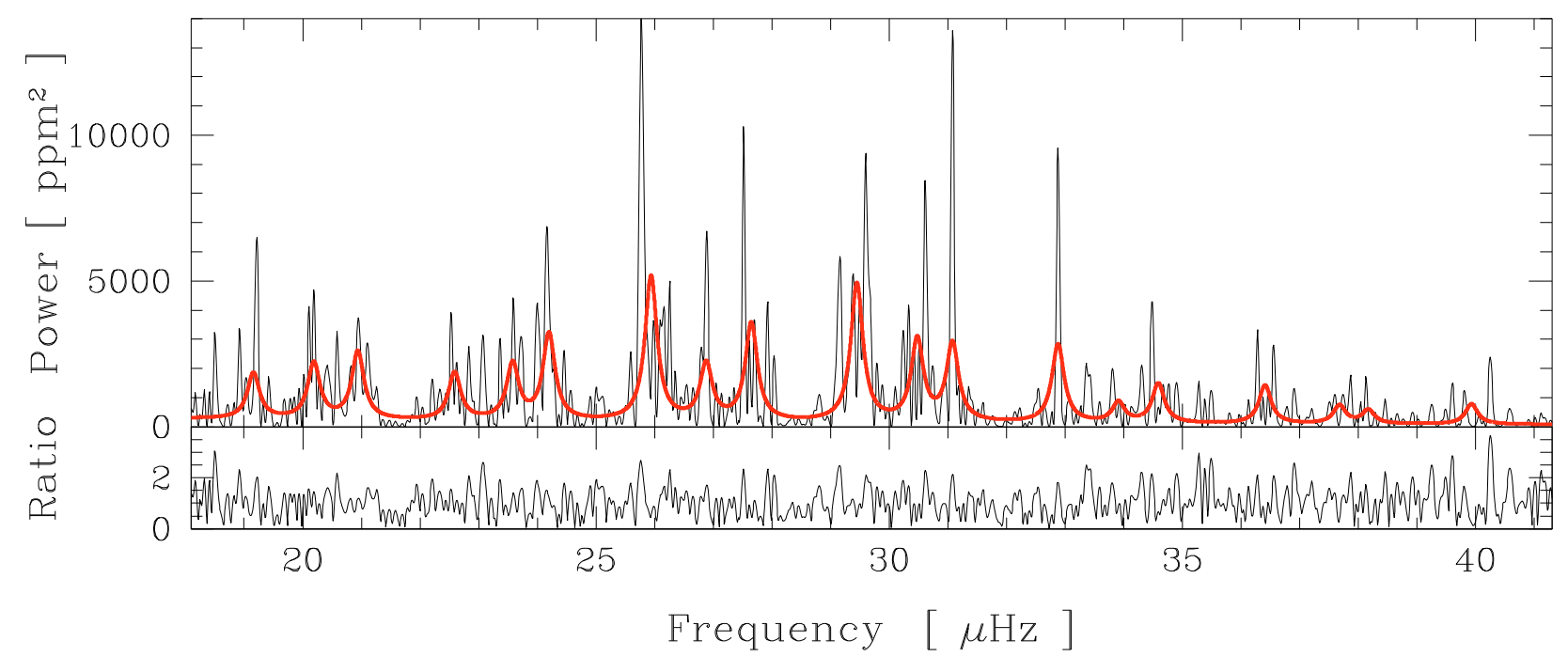

Fig. 7. Top: Lorentzian fit (thick red line) of the observed power spectrum assuming the same lifetime for all the oscillation modes. Bottom: ratio of the observed spectrum over the Lorentzian fit (in amplitude). As expected, this ratio does not show any correlation with the fit.

two modes will however be more uncertain. The result is listed in Table 1. The formal uncertainties associated with MLE are well understood, as explained by Libbrecht (1992) and Toutain \& Appourchaux (1994). The echelle diagram with the nineteen identified modes is shown in Fig. 8. At higher and lower frequency (above $40 \mu \mathrm{Hz}$ and below $19 \mu \mathrm{Hz}$ ), the amplitude of the modes is either too small or the noise too high to unambiguously identify additional modes. The ratio of the observed power spectrum to the fit is shown in Fig. 7: it appears to be pure noise and has a mean value of 1 . Moreover, no correlation was found between the amplitude of this ratio and the amplitude of the fit. We note that the non-radial modes are as well aligned as radial or non-mixed modes. However, the separation between $\ell=1$ and $\ell=0$ modes is not fully compatible with the asymptotic relation. The $\ell=1$ modes are indeed too far to the right in the echelle diagram.

The large and small separations are shown in Fig. 9. The mean large separation has a value of $\Delta v=3.47 \pm 0.12 \mu \mathrm{Hz}$, and the values for different degrees $\ell, \Delta v_{\ell}$, are: $\Delta v_{0}=3.45 \pm 0.12 \mu \mathrm{Hz}, \Delta v_{1}=3.46 \pm 0.07 \mu \mathrm{Hz}$, and $\Delta v_{2}=3.50 \pm 0.19 \mu \mathrm{Hz}$. We can identify a small oscillation of the large spacing that varies with frequency, which is a clear signature of the second helium ionization zone (see e.g., Monteiro \& Thompson 1998). The small separation has a mean value of $\delta v_{02}=0.65 \pm 0.10 \mu \mathrm{Hz}$ and seems to decrease with frequency. The small value of the frequency difference between these modes make their frequency determination more uncertain than that of $\ell=1$ modes, which are not affected by neighbouring modes.

\subsection{Oscillation amplitudes}

The fit of the Lorentzian profiles to the power spectrum infers the height of all oscillation modes. Since the modes are resolved and because of the normalization of the power spectrum, the RMS amplitude is measured to be (see Baudin et al. 2005)

$A=\sqrt{H \pi \Gamma}$,

where $H$ and $\Gamma$ are the height and width (FWHM) of the Lorentzian function, respectively, in the power density spectrum. The amplitudes are in the range of $35-115 \mathrm{ppm}$ (see Fig. 10).

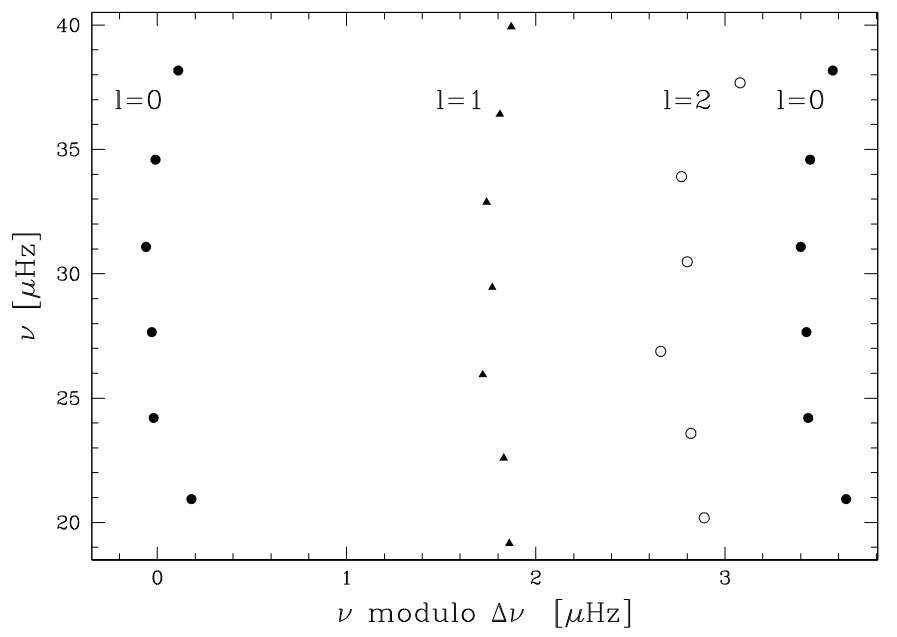

Fig. 8. Echelle diagram of identified modes with a large separation of $\Delta v_{0}=3.45 \mu \mathrm{Hz}$. The modes $\ell=0(\bullet), \ell=1(\boldsymbol{\Delta})$, and $\ell=2$ (o) follow ridges, but $\ell=1$ modes are situated too far to the right to follow the asymptotic relation.

The error bars are derived from the Hessian matrix and the correlation between the height and width has been taken into account (Toutain \& Appourchaux 1994, Appourchaux, private communication). We note that the amplitudes of the $\ell=1$ modes are the highest.

As noticed by Kjeldsen et al. (2005), measurements made on different stars with different instruments using different techniques, in different spectral lines or bandpasses, have different sensitivity to the oscillations. It is thus important to derive a bolometric amplitude that is independent of the instrument used. We computed the maximum bolometric amplitude of the $\ell=0$ modes, because their visibility coefficients do not depend on the inclination of the star. According to Michel et al. (2009), who derived the CoRoT response for radial modes, radial-mode amplitudes of HR 7349 must be divided by 1.16 to obtain the bolometric amplitudes. We find that $A_{\mathrm{bol}, \ell=0, \max }=81 \mathrm{ppm}$, which corresponds to 32 times the solar value (Michel et al. 2009). The scaling laws for both the large separation and the frequency of the maximum amplitude (Kjeldsen \& Bedding 1995), coupled 
Table 1. Frequency and amplitude of identified modes.

\begin{tabular}{ccccc}
\hline \hline $\begin{array}{c}\text { Degree } \\
\ell\end{array}$ & $\begin{array}{c}\text { Frequency } \\
\mu \mathrm{Hz}\end{array}$ & $\begin{array}{c}\text { Mode height } \\
\mathrm{ppm}^{2} * 1000 / \mu \mathrm{Hz}\end{array}$ & $\begin{array}{c}S / N \\
\text { Amplitude } \\
\mathrm{ppm}\end{array}$ \\
\hline 0 & $20.94(7)$ & 7.8 & 10.8 & $79 \pm 15$ \\
0 & $24.20(7)$ & 10.0 & 17.6 & $89 \pm 15$ \\
0 & $27.65(6)$ & 11.3 & 24.8 & $94 \pm 16$ \\
0 & $31.08(6)$ & 9.0 & 23.7 & $84 \pm 14$ \\
0 & $34.59(7)$ & 4.6 & 14.0 & $60 \pm 11$ \\
0 & $38.17(9)$ & 1.6 & 5.6 & $35 \pm 8$ \\
1 & $19.16(7)$ & 5.3 & 6.2 & $65 \pm 14$ \\
1 & $22.59(7)$ & 5.5 & 8.6 & $66 \pm 13$ \\
1 & $25.94(5)$ & 16.8 & 33.1 & $115 \pm 19$ \\
1 & $29.45(5)$ & 16.0 & 38.8 & $112 \pm 18$ \\
1 & $32.88(5)$ & 9.1 & 26.0 & $85 \pm 14$ \\
1 & $36.41(6)$ & 4.4 & 14.6 & $59 \pm 11$ \\
1 & $39.93(6)$ & 2.3 & 8.8 & $43 \pm 9$ \\
2 & $20.19(8)$ & 6.5 & 8.4 & $71 \pm 14$ \\
2 & $23.58(7)$ & 6.5 & 10.9 & $71 \pm 13$ \\
2 & $26.88(7)$ & 6.5 & 13.7 & $72 \pm 13$ \\
2 & $30.48(6)$ & 9.5 & 24.1 & $86 \pm 15$ \\
2 & $33.91(8)$ & 2.3 & 7.0 & $43 \pm 9$ \\
2 & $37.68(8)$ & 2.1 & 7.3 & $41 \pm 8$ \\
\hline
\end{tabular}

Notes. The uncertainties in the last given digit of the frequencies are noted in parentheses. For the $S / N$ estimates (see Barban et al. 2007), the signal is taken to be the height of the fitted Lorentzian profile and the noise is determined according to the procedure described in Sect. 4.1.

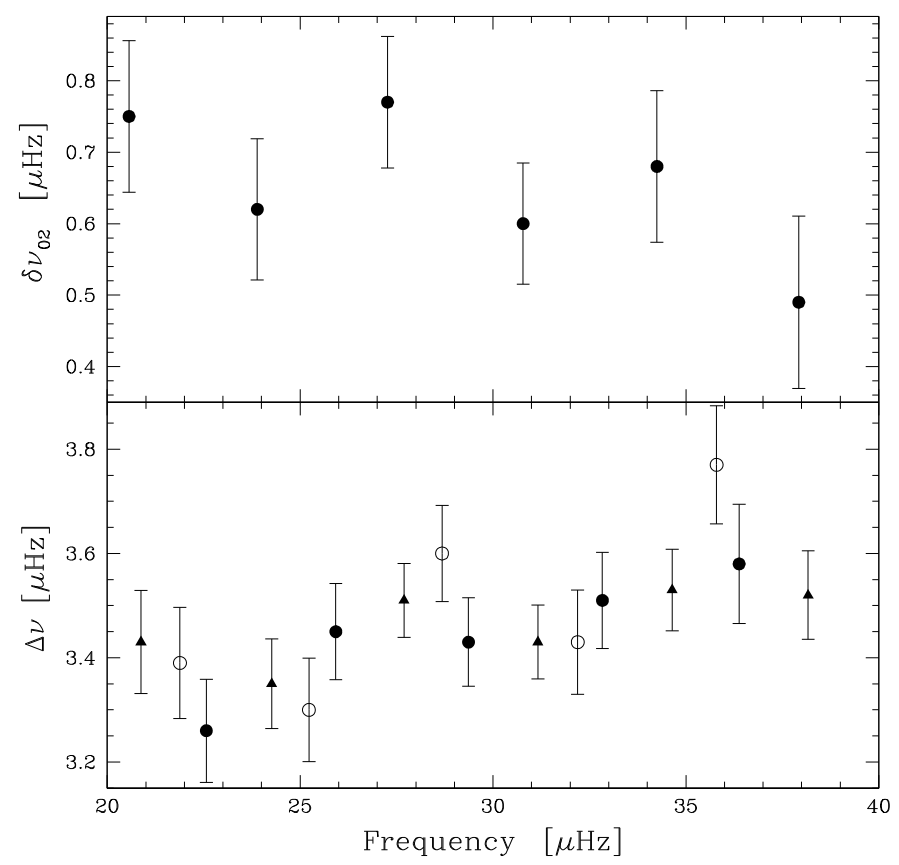

Fig. 9. Top: small spacing versus frequency. Bottom: large spacing versus frequency. The variations of the large separation with frequency show a clear oscillation. The symbols used are the same as for Fig. 8.

with the non-asteroseismic constraints, infer a mass for HR 7349 of about $1.2 M_{\odot}$ The derived amplitude is in good agreement with a scaling function $(L / M)^{s} / \sqrt{T_{\text {eff }} / T_{\text {eff, } \odot}}$, with $s$ close to 0.8 , which is in-between the values given by Samadi et al. (2007; $s=0.7)$ and Kjeldsen \& Bedding (1995; $s=1)$.

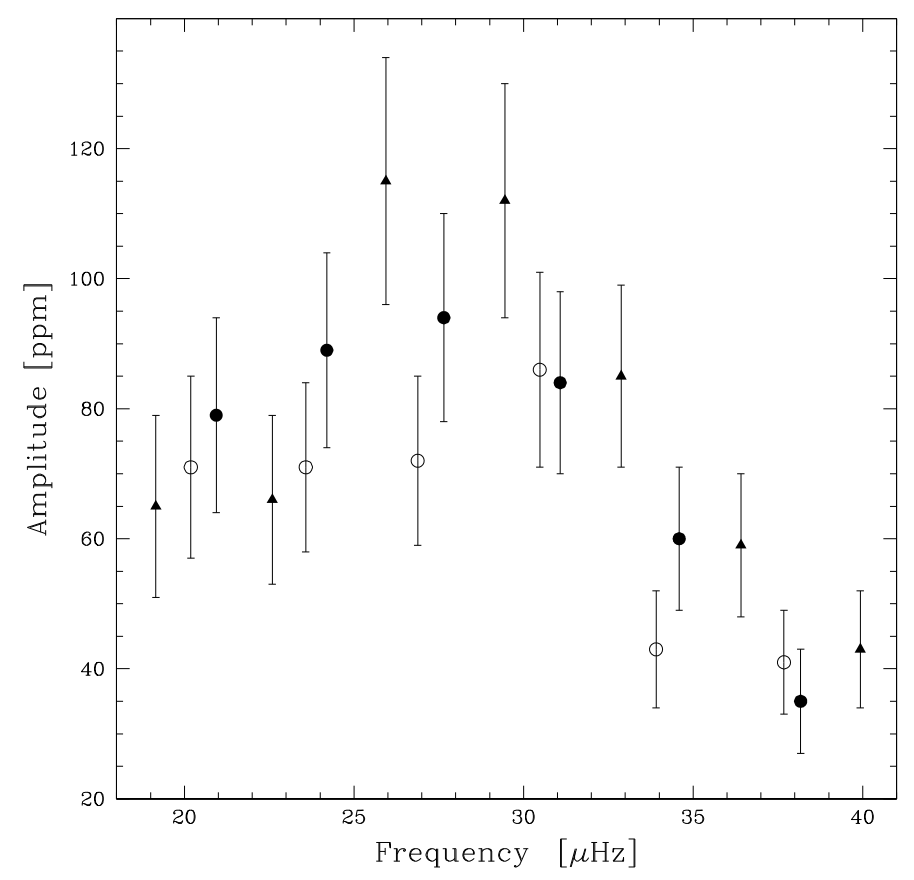

Fig. 10. Amplitude of the oscillation modes versus frequency. The symbols used are the same as for Fig. 8.

\section{Conclusion}

The red giant star HR 7349 has been observed for about 156 days by the CoRoT satellite. These observations have yielded a clear detection of p-mode oscillations. As already mentioned by De Ridder et al. (2009), non-radial modes are observed in red giants. Nineteen identifiable modes of degree $\ell=0$ to $\ell=2$ appear in the power spectrum between 18 and $42 \mu \mathrm{Hz}$ with average large and small spacings of 3.47 and $0.65 \mu \mathrm{Hz}$, respectively, and a maximum bolometric amplitude of $81 \mathrm{ppm}$. We note that the amplitude of $\ell=1$ modes is even larger than that of the radial modes.

All modes of the same degree are aligned in the echelle diagram, which is a sign of modes that follow the asymptotic relation. However, the separation between $\ell=1$ and $\ell=0$ modes is not fully compatible with this asymptotic relation. All frequency patterns are theoretically expected for red giants (Dupret et al. 2009), from very complex to regular: our observations correspond to a red giant for which the radiative damping of nonradial modes is large and only radial and non-radial modes completely trapped in the envelope can be observed.

By fitting Lorentzian profiles to the power spectrum, it has also been possible to unambiguously derive, for the first time for a red giant, a mean line-width of $0.25 \mu \mathrm{Hz}$ corresponding to a mode lifetime of 14.7 days. This lifetime is in agreement with the scaling law $T_{\mathrm{eff}}^{-4}$ suggested by Chaplin et al. (2009), although is a little too long. This relation however has yet to be verified for a larger number of red giants with different physical properties. The theoretical study of this red giant, including asteroseismic and non-asteroseismic constraints, will be the subject of a second paper.

Acknowledgements. F.C. is a postdoctoral fellow of the Fund for Scientific Research, Flanders (FWO). AM is a postdoctoral researcher of the "Fonds de la recherche scientifique" FNRS, Belgium. T.K. is supported by the Canadian Space Agency and the Austrian Science Found (FWF). The research leading to these results has received funding from the Research Council of K.U. Leuven under grant agreement GOA/2008/04, from the Belgian PRODEX Office under 
F. Carrier et al.: Non-radial oscillations in the red giant HR 7349 measured by CoRoT

contract C90309: CoRoT Data Exploitation, and from the FWO-Vlaanderen under grant O6260. We thank T. Appourchaux for helpful comments.

\section{References}

Aigrain, S., Favata, F., \& Gilmore, G. 2004, A\&A, 414, 1139

Alonso, A., Arribas, S., \& Martinez-Roger, C. 1999, A\&AS, 140, 261

Andersen, B., Appourchaux, T., \& Crommelnynck, D. 1998, in Sounding solar and stellar interiors, ed. Provost, \& Schmider, 181, 147

Appourchaux, T., Gizon, L., \& Rabello-Soares, M. C. 1998, A\&AS, 132, 107

Appourchaux, T., Michel, E., Auvergne, M., et al. 2008, A\&A, 488, 705

Arenou, F., Grenon, M., \& Gomez, A. 1992, A\&A, 258, 104

Auvergne, M., Bodin, P., Boisnard, L., et al. 2009, A\&A, 506, 411

Baglin, A. 2006, ESA SP-1306, The CoRoT mission, ed. M. Fridlund, A. Baglin, J. Lochard and L. Conroy (Noordwijk, The Nederlands)

Barban, C., De Ridder, J., Mazumdar, A., et al. 2004, ESA SP-559, SOHO 14 Helio- and Asteroseismology, ed. D. Danesy, 113

Barban, C., Matthews, J. M., De Ridder, J., et al. 2007, A\&A, 468, 1033

Baudin, F., Samadi, R., Goupil, M.-J., et al. 2005, A\&A, 433, 349

Bedding, T. R., \& Kjeldsen H. 2007, CoAst, 150, 106

Burki, G., et al. 2008, http : //obswww . unige.ch/gcpd/ph13.html

Carrier, F., Eggenberger, P., \& Leyder, J. C. 2008, JPHCS, 118, 2047

Chaplin, W. J., Appourchaux, T., Baudin, F., et al. 2006, MNRAS, 369, 985

Chaplin, W. J., Houdek, G., Karoff, C., et al. 2009, A\&A, 500, L21

Christensen-Dalsgaard, J. 2004, Sol. Phys., 220, 137
Christensen-Dalsgaard, J., Bedding, T. R., \& Kjeldsen, H. 1995, ApJ, 443, L29 Dupret, M.-A., Belkacem, K., Samadi, R., et al. 2009, A\&A, 506, 57 De Ridder, J., Barban, C., Carrier, F., et al. 2006, A\&A, 448, 689 De Ridder, J., Barban, C., Baudin., F., et al. 2009, Nature, 459, 398 Fernandes, J., \& Monteiro, M. J. P. F. G. 2003, A\&A, 399, 243 Flower, P. 1996, ApJ, 469, 355

Frandsen, S., Carrier, F., Aerts, C., et al. 2002, A\&A, 394, L5 Kjeldsen, H., \& Bedding, T. 1995, A\&A, 293, 87

Kjeldsen, H., Bedding, T., Butler, R. P., et al. 2005, ApJ, 635, 1281

Harvey, J. 1985, in Future Missions in Solar, Heliospheric, \& Space Plasma Physics, ed. Rolfe \& Battrick, ESA SP, 235, 199

Hekker, S., \& Meléndez, J. 2007, A\&A, 475, 1003

Hekker, S., Kallinger, T., Baudin, F., et al. 2009, A\&A, 506, 465

Lejeune, T., Cuisinier, F., \& Buser, R. 1998, A\&AS, 130, 65

Libbrecht, K. G. 1992, ApJ, 387, 712

Michel, E., Samadi, R., Baudain, F., et al. 2009, A\&A 495, 979

Monteiro, M. J. P. F. G., \& Thompson, M. J. 1998, IAU Symp., 185, 317

Samadi, R., Georgobiani, D., Trampedach, R., et al. 2007, A\&A, 463, 297

Santos, N. C., Mayor, M., Naef, D., et al. 2002, A\&A, 392, 215

Schou, J. 1992, Ph.D. Thesis (Denmark: Aarhus Universitet)

Stello, D., Kjeldsen, H., Bedding, T. R., \& Buzasi, D. 2006, A\&A, 448, 709

Tarrant, N. J., Chaplin, W. J., Elsworth, Y. P., et al. 2008, CoAst, 157, 92

Tassoul, M. 1980, ApJS, 43, 469

Toutain, T., \& Appourchaux, T. 1994, A\&A, 289, 649

van Leeuwen, F. 2007, A\&A, 474, 653 